\title{
ASOCIACIÓN DEL HÁBITO DE FUMAR Y LA MALA HIGIENE ORAL CON LA ENFERMEDAD PERIODONTAL EN PACIENTES QUE ACUDEN A LA CONSULTA ODONTOLÓGICA DE LA UNIVERSIDAD SANTO TOMÁS
}

\author{
${ }^{1}$ José Duvian Angulo C., ${ }^{1}$ Luís Carlos Márquez, ${ }^{1}$ Martha Xiomara Navas C., ${ }^{2}$ Luz Elena Archila A. \\ ${ }^{1}$ Estudiante de X semestre, F. de Odontología, U. Santo Tomás. ${ }^{2}$ Odontóloga, U. Santo Tomás; Especialista en Periodoncia y \\ Medicina Oral, U. El Bosque, Docente, U. Santo Tomás.
}

Autor responsable de correspondencia: Martha Xiomara Navas C.

Correo electrónico: xiomync@hotmail.com

\section{RESUMEN}

Objetivo: Establecer la asociación de la enfermedad periodontal con el hábito de fumar en personas que acuden a la consulta odontológica en las clínicas de la Universidad Santo Tomás.

Materiales y métodos: Se realizó un estudio observacional analítico de corte transversal. El universo lo constituyeron 760 pacientes entre 20 y 60 años y la muestra 178 personas. Ochenta y nueve fumadores y 89 no fumadores, seleccionados mediante muestreo aleatorio simple; se consideró como variable de salida la presencia de enfermedad periodontal y explicatorias las otras variables recolectadas. El plan de análisis estadístico univariado incluyó el cálculo de medidas de resumen; para el bivariado se aplicaron test de Chi cuadrado o Exacto de Fisher. Para el multivariado se aplicó una regresión binomial, considerando como variable de salida la presencia de enfermedad periodontal, se calcularon RR (razón de prevalencia) y sus respectivos intervalos de confianza.

Resultados: Se observó la presencia de al menos un marcador periodontal en el $87 \%$ de las personas evaluadas. Hubo una asociación casi dos veces mayor de presentar enfermedad periodontal en pacientes fumadores en comparación con los no fumadores y un riesgo relativo de cuatro veces mayor en personas fumadoras con regular higiene oral.

Conclusión: Se estableció que el hábito de fumar es un factor significativo en el desarrollo y la progresión de la enfermedad periodontal, que el sangrado gingival está disminuido en estos pacientes y que aspectos como la prevención, la educación y la motivación de la higiene oral son fundamentales para controlar o evitar que se desarrolle esta patología. [Ángulo JD, Márquez LC, Navas MX, Archila LE. Incremento de la asociación del hábito de fumar y la mala higiene oral con la enfermedad periodontal en pacientes que acuden a la consulta odontológica de la Universidad Santo Tomás. Revista Ustasalud Odontología 2007; 6: 104 - 111]

Palabras clave: Enfermedad periodontal, Hábito de fumar, Higiene oral.

\section{ASSOCIATION OF THE SMOKE HABIT AND THE BAD ORAL HYGIENE WITH PERIODONTAL DISEASE IN PATIENTS WHO ATTEND THE DENTAL CLINICS OF SANTO TOMAS UNIVERSITY}

\section{ABSTRACT}

Objective: To determine the relationship between periodontal disease and smoking habit in patients, that attended the dental clinics in la Santo Tomas University.

Materials and methods: An analytical cross section observational study was made. The universe constituted by 760 patients between 20 and 60 years old and the sample by 178 patients, 89 smokers and 89 nonsmokers selected by means of simple random sampling. The simple statistical analysis included the calculation of summary measures; in the bivaried analysis test of square Chi or Fisher Exact test were applied. By the multivaried analysis a binomial regression was applied, that considered as an exit variable the presence of Periodontal Disease, and RR and its respective confidence intervals were calculated.

Results: The presence of a periodontal marker was observed at least in $87 \%$ of the evaluated patients; There was a greater association almost two times to present periodontal disease in smoker patients in comparison with the nonsmokers and greater a relative risk of four times in smoker people with index of regular hygiene.

Conclusion: It was established the habit to smoke is a significant factor in the development and the progression of the periodontal diseases, that the gingival bled one is diminished in these patients and who aspects like the prevention, the education and the motivation of the oral hygiene are fundamental so that this pathology is not developed

Key words: Periodontal diseases, Smoke habit, Oral hygiene. 


\section{INTRODUCCIÓN}

La Enfermedad Periodontal es una patología infecciosa multifactorial que afecta los dientes, comprende una variedad de estados clínicos caracterizados por la inflamación y / o destrucción de las estructuras de soporte de los dientes. La etiología de la enfermedad periodontal no se conoce con exactitud, sin embargo, muchas de sus manifestaciones se asocian con la presencia de bacterias patógenas, la respuesta inmune del huésped, hábitos y estrés. ${ }^{1}$

El tabaco es originario de América. La planta Nicotiana tabacum fue conocida por los exploradores europeos al ser ofrecida como regalo a Cristóbal Colón en las Indias Occidentales en $1942 .{ }^{2}$ En Colombia se descubrió su uso, entre otros para mordedura de serpiente (Orinoquía), mezclado con hojas de coca para detener hemorragias (Indígenas Paeces) y en general los rituales mágicos de adivinación o de iniciación de las mamas, piaches, brujos o shamanes. ${ }^{2}$

Se ha comprobado que el fumar se relaciona directamente con la incidencia y prevalencia de diferentes problemas médicos, entre los que se incluye el cáncer, enfermedades pulmonares, cardiovasculares y gastrointestinales. Durante varios años de estudios científicos se ha comprobado que los factores de riesgo para las enfermedades periodontales incluyen género, raza, edad y la condición del fumador. ${ }^{3}$

La prevalencia de periodontitis es mayor en los fumadores que en los no fumadores y el hueso alveolar es más susceptible a reabsorción en los fumadores, ${ }^{4}$ esta pérdida ósea se encuentra en estrecha relación con la duración de la exposición al tabaco así como también se encuentra relacionado con la pérdida de piezas dentarias. Se ha encontrado una fuerte relación entre fumar y la pérdida de hueso alveolar así como también con la prevalencia de periodontitis severa. $^{5}$

Existe una relación entre el cigarrillo y varias formas de enfermedad periodontal. La gingivitis y la periodontitis son causadas por bacterias; fumar es un factor de riesgo en la iniciación y propagación de la enfermedad periodontal. Dicho hábito tam- bién se relaciona con la acumulación de cálculo, que puede producir la recesión gingival y el incremento en la profundidad de las bolsas periodontales. La nicotina, un alcaloide, considerado como el compuesto farmacológicamente activo del tabaco, y que determina el comportamiento aditivo del fumador, activa el sistema nervioso simpático e incrementa el ritmo cardíaco y genera vasoconstricción. $^{2}$

La nicotina y sus metabolitos como la cotidina, cuando son absorbidos por los tejidos, se unen a receptores específicos e inducen la liberación de adrenalina que produce una vasoconstricción periférica y reduce el drenaje de los catabólitos residuales, altera el pH y el potencial de óxido reducción de los tejidos. ${ }^{6}$

Los signos clínicos de la gingivitis como el sangrado gingival e inflamación son más difíciles de observar en los pacientes fumadores. En éstos se encuentra aumentada la temperatura subgingival que se considera como un indicador de enfermedad periodontal, tal incremento promueve el desarrollo de bacterias como P intermedia, Peptostreptococcus, Porphyromonas gingivalis, A. actinomycetemcomitans. $^{\text {? }}$

Según el tercer estudio nacional de salud bucal en el año 1998 (ENSAB III Tomo VII), la presencia de al menos un marcador periodontal se observó en el 92.4\% de las personas, de éstas el $8.8 \%$ presentó sangrado al sondaje, el 53.3\% presentó sangrado y cálculos simultáneamente; las bolsas (menores de $6 \mathrm{~mm}$ ) se diagnosticaron en el $26.5 \%$ y las profundas (de 6 y más $\mathrm{mm}$ ) en el 3,8\% (95\% L.C. 3.78 $-3.81)^{8}$

En este estudio, se determinó la asociación del hábito de fumar con la enfermedad periodontal en personas que asistieron a consulta odontológica en las clínicas odontológicas de la USTA de Floridablanca.

\section{MATERIALES Y MÉTODOS}

Se realizó un estudio observacional analítico de corte transversal, ${ }^{9-11}$ cuya población estuvo conformada por 760 pacientes entre 20 y 60 años que acudieron a las clínicas odontológicas de la USTA

105 
en el campus universitario de Floridablanca que presentan la condición de fumadores y no fumadores.

El tamaño de la muestra se calculó mediante la rutina simple size del paquete estadístico Epi Info $6.04^{12}$ se consideraron las siguientes especificaciones de diseño para un estudio analítico de corte transversal: nivel de confianza: $95 \%$, poder: $80 \%$, con una relación expuesto no expuesto 1-1, con una frecuencia de enfermedad periodontal de acuerdo a lo establecido en ENSAB III de $50.9 \%,{ }^{8}$ con una frecuencia de enfermedad periodontal en no fumadores $28.6 \%$, de acuerdo a la prueba piloto; con un RR de 1.75 y un OR de 2.51; por lo cual el tamaño de la muestra estuvo constituido por 178 personas de los cuales 89 fueron fumadores y 89 no fumadores. La selección se hizo por muestro probabilístico de tipo aleatorio simple.

Se incluyó en el estudio todo paciente activo de las clínicas odontológicas de la Universidad Santo Tomás en el campus universitario de Floridablanca fumadores y no fumadores, pacientes con edades entre 20 y 60 años. Se consideraron como criterios de exclusión las mujeres en estado de embarazo, pacientes menores de 30 años con periodontitis agresiva, pacientes con tratamiento de ortodoncia, terapia antibiótica y pacientes comprometidos sistémicamente.

En esta investigación se aplicó un instrumento tipo encuesta autodiligenciada, en la que se registraron variables sociodemográficas, género, edad, estrato socioeconómico, nivel educativo; se recolectaron variables como fumar, frecuencia de consumo de cigarrillos, estado de salud periodontal (de acuerdo a lo establecido para el Índice Comunitario Periodontal de Necesidad de Tratamiento, ICPNT ${ }^{8}$ movilidad dental, higiene oral, sensibilidad dental, y el índice de extensión y severidad.

Previa a la recolección de datos se efectuó una prueba piloto con 15 pacientes que acudieron a las clínicas odontológicas de la Universidad Santo Tomás en Floridablanca, a partir de ésta se modificó el instrumento, se estandarizaron los procedimientos y se definieron los tiempos para el desarrollo de la investigación y el diligenciamiento de la encuesta.
Inicialmente, se procedió a la selección, ubicación, coordinación de la aplicación de la encuesta que se dividió en dos fases, el autodiligenciamiento y la otra parte el examen clínico que se realizaba por parte de un investigador entrenado; previa a estas dos fases se obtuvo el respectivo consentimiento informado.

Los datos se procesaron en Excel $^{13}$ por duplicado, se exportó a EPI-INFO $6.04^{12}$ y mediante la rutina validate se corroboró la calidad de la digitación. Finalmente se exportó al paquete estadístico STATA $9.1^{14}$ para su correspondiente procesamiento y análisis. El plan de análisis incluyó un análisis univariado que describe las variables mediante medidas de tendencia central y dispersión según la naturaleza y el bivariado en el que se estableció la asociación entre condición con cada variable mediante test de chi ${ }^{2}$ y exacto de Fisher y para las variables cuantitativas se aplicaron pruebas de $t$ de student o de rangos de Wicolxon, de acuerdo a la distribución de los datos. ${ }^{15,16}$

Para el análisis multivariado se aplicó un análisis de regresión binomial que estimó los RR: (Riesgos Relativos: Razón de prevalencia) y sus respectivos intervalos de confianza del $95 \%$. Se definió como variable de salida Enfermedad Periodontal y como variables explicatorios las otras variables recolectadas. ${ }^{17,18}$ Se analizaron por separado cada una de estas variables en el análisis de regresión binomial bivariado inicial. A partir de éste se seleccionaron para el modelo final las variables que obtuvieran probabilidad igual o inferior a $\mathrm{p}=0.25 \mathrm{y}$ se forzaron en el modelo aquellas que se consideraron biológicamente significativas. ${ }^{19}$ Todo el análisis se realizó con un nivel de significancia de Alfa $(\alpha)=$ 0.05 .

De acuerdo con la resolución 008430 de 1993 emitida por el Ministerio de Salud en la cual se establecen las normas científicas, técnicas y administrativas para investigaciones en salud y que en el artículo 11 , literal b, esta investigación se considera de riesgo mínimo, ya que se recolectaron datos a través de procedimientos odontológicos de rutina. ${ }^{20}$ 


\section{RESULTADOS}

\section{Aspectos sociodemográficos}

Análisis univariado

Se evaluaron 178 pacientes, con un promedio de edad de $35.3 \pm 11.0$ y rango entre 20 y 60 años, de los cuales el 50\% (89) pertenecían al grupo de fumadores y el grupo restante al de no fumadores. El 54.5\% (97) pertenecían al género masculino. El 43.3\% (77) de los evaluados pertenecían al estrato 2 , seguido por el estrato 3 con un 40.5\% (72) de los sujetos analizados. Al referirnos al nivel educativo el 48.9\% (87) cursaban o habían cursado el bachillerato y el $1.1 \%$ (2) no habían tenido estudios educativos (Tabla 1).

\section{Análisis bivariado}

$\mathrm{Al}$ analizar la población evaluada de acuerdo a las variables sociodemográficas se encontró que del total de hombres evaluados $88.2 \%(15)$ tenían bolsa de $6 \mathrm{~mm}$, mientras que del total de mujeres sólo el $11.8 \%$ (2) registraban igual condición; sin embargo, para esta variable no se observaron asociaciones estadísticamente significativas $(p=0.053)$.

Al analizar por grupo de edad se observó en el grupo de 45 años y más, sólo el 4.4\% (1) de ellos fue catalogado como sano mientras que el $58.8 \%$ (10) de las personas en este grupo de edad registró bolsas de $6 \mathrm{~mm}$. Se hizo evidente una asociación estadísticamente significativa entre edad y condición periodontal $(\mathrm{p}<0.001)$; igual fenómeno se hizo evidente para estrato socioeconómico y nivel educativo. Para la primera en el total de personas catalogadas en estrato 3 , el $78.3 \%$ (18) se clasificó como sana y sólo el $11.8 \%$ (2) presentó bolsas de $6 \mathrm{~mm}$. Para la segunda, es importante resaltar que del total de personas con nivel universitario el $52.2 \%$ (12) estaban sanas, sólo el 4.4\% (1) se diagnóstico con bolsas de 4 a $5 \mathrm{~mm}$ y ninguna registró bolsas de $6 \mathrm{~mm}$. (Tabla 1).

\section{Aspectos relacionados con el examen clínico}

Análisis univariado

La presencia de al menos un marcador periodontal se observó en el $87.1 \%$ (155) de la población analizada; de éstas, el 10.1\% (18) presentó sangrado al sondaje, $54.5 \%$ (54.5) presentó cálculos, 12.9\% (23) bolsa de 4 a 5mm, y $9.6 \%$ (17) bolsas de $6 \mathrm{~mm}$. Adicionalmente, el 8.4\% (15) de los evaluados presentaron sensibilidad dental, el $15.2 \%$ (27) presentaron movilidad grado I y el 1.7\%(3) movilidad grado II; el 41\% (73) registraron índice de placa regular y el $33.7 \%$ (60) índice malo (Tabla 2).

\section{Análisis bivariado}

En el total de personas catalogadas como sanas, el $100 \%$ (23) no reportaban tener movilidad dentaria mientras que sólo el 5.9\% (1) del total de clasificadas con bolsas de $6 \mathrm{~mm}$ no registraban movilidad. Además, del total de personas catalogadas como periodontalmente sanas, el 100\% registraban buena higiene oral, mientras que del total de sujetos con bolsas de $6 \mathrm{~mm}$, el 88.2\% (15) reportaban mala higiene bucal; tanto para movilidad dentaria como para nivel de placa dentobacteriana se observaron asociaciones estadísticamente significativas $(\mathrm{p}<0.0001)$ (Tabla 2)

\section{Análisis Multivariado}

En el modelo de regresión binomial no ajustado para la enfermedad periodontal se encontraron como factores asociados a tener enfermedad periodontal el hábito de fumar (R.R. 1.97 IC 95\% 1.05 - 3.68); la edad (R.R.1.03 IC 95\% 1.0 - 1.1); el nivel educativo la primaria (R.R. 1.81 IC 95\% 0); la higiene regular (R.R. 2.77 IC 95\% 1.79 - 4.27) y el índice malo (R.R. 2.77 IC 95\% 1.79 - 4.27); todas éstas variables mostraron significancia estadísticamente con valor $P$ de $0.003,0.011$ y $<0.001$ al referirlas en el orden anteriormente mencionado (Tabla 3).

El modelo de regresión binomial ajustado para todas las variables mostró que fumar con R.R. de 3.35 IC95\% (1.61-6.95); al igual que el tener un índice de higiene regular con R.R. de 4.33 IC95\% (1.73-10.81) están asociados a presentar enfermedad periodontal. Existe significancia estadística para éstas variables con valor $\mathrm{p}$ de 0.001 para la primera y de $\mathrm{p}=0.002$ para la segunda (Tabla 4). 
Tabla 1. Descripción de las variables sociodemográficas en forma global según la enfermedad periodontal.

\begin{tabular}{|c|c|c|c|c|c|c|c|}
\hline \multirow[b]{2}{*}{ VARIABLE } & \multirow[b]{2}{*}{ Global } & \multicolumn{6}{|c|}{ CONDICIÓN PERIODONTAL } \\
\hline & & Sano & Sangrado & Cálculo & $\begin{array}{l}\text { Bolsa de } \\
4 \text { a } 5 \mathrm{~mm}\end{array}$ & $\begin{array}{c}\text { Bolsa de } 6 \\
\text { mm }\end{array}$ & $\mathbf{P}^{*}$ \\
\hline Global & $178(100)$ & $23(12.9)$ & $18(10.1)$ & $97(54.5)$ & $23(12.9)$ & $17(9.6)$ & \\
\hline \multicolumn{8}{|l|}{ GÉNERO } \\
\hline Mujer & $81(45.5)$ & $10(43.4)$ & $10(55.6)$ & $47(48.5)$ & $12(52.6)$ & $2(11.8)$ & $0.053+$ \\
\hline Hombre & $97(54.5)$ & $13(56.5)$ & $8(44.4)$ & $50(51.6)$ & $11(47.8)$ & $15(88.2)$ & \\
\hline \multicolumn{8}{|l|}{ EDAD } \\
\hline 20 a 24 años & $37(20.8)$ & 9(39.1) & $3(16.7)$ & $25(25.8)$ & --- & --- & \\
\hline 25 a 29 años & $29(16.3)$ & $8(34.8)$ & $6(33.3)$ & 11(11.34) & $4(17.4)$ & --- & \\
\hline 30 a 34 años & $29(16.3)$ & $2(8.7)$ & $3(16.7)$ & $18(18.6)$ & $6(26.1)$ & -- & $<0.001+$ \\
\hline 35 a 39 años & 18(10.1) & $1(4.35)$ & $2(11.1)$ & $10(10.3)$ & $3(13.0)$ & $2(11.8)$ & \\
\hline 40a44 años & 18(10.1) & $2(8.7)$ & $1(5.6)$ & $9(9.3)$ & $1(4.4)$ & $5(29.4)$ & \\
\hline 45 a 59 años & $47(26.4)$ & $1(4.35)$ & $3(16.7)$ & $24(24.7)$ & 9(39.1) & $10(58.8)$ & \\
\hline \multicolumn{8}{|c|}{ ESTRATO SOCIOECONÓMICO } \\
\hline 1 & $21(11.8)$ & --- & $1(5.6)$ & $17(17.53)$ & $3(13.14)$ & --- & \\
\hline 2 & $77(43.3)$ & 1(4.359 & $6(33.33)$ & $39(40.21)$ & $16(69.6)$ & $15(88.2)$ & $<0.001+$ \\
\hline 3 & $72(40.5)$ & $18(78.3)$ & $10(55.6)$ & $38(39.2)$ & $4(17.4)$ & $2(11.8)$ & \\
\hline 4 & $8(4.5)$ & $4(17.4)$ & $1(5.6)$ & $3(3.1)$ & --- & -- & \\
\hline \multicolumn{8}{|l|}{ NIVEL EDUCATIVO } \\
\hline No tiene estudios & $2(1.1)$ & --- & --- & $2(2.1)$ & --- & --- & \\
\hline Primaria & $49(27.5)$ & -- & $3(16.7)$ & $22(22.7)$ & $9(39.3)$ & $15(88.12)$ & $<0.001+$ \\
\hline Bachillerato & $87(48.9)$ & $11(47.8)$ & $9(50.0)$ & $52(53.6)$ & $13(56.5)$ & $2(11.8)$ & \\
\hline Universidad & $40(22.5)$ & $12(52.2)$ & $6(33.3)$ & $21(21.65)$ & $1(4.4)$ & --- & \\
\hline
\end{tabular}

Tabla 2. Descripción de las variables relacionadas con el examen clínico en forma global y según la condición periodontal.

\begin{tabular}{|c|c|c|c|c|c|c|c|}
\hline \multirow{3}{*}{ VARIABLES } & \multicolumn{7}{|c|}{ CONDICIÓN PERIODONTAL } \\
\hline & Global & Sano & Sangrado & Cálculo & $\begin{array}{l}\text { Bolsa de } 4 \text { a } \\
\quad 5 \mathrm{~mm}\end{array}$ & $\begin{array}{c}\text { Bolsa de } 6 \\
\text { mm }\end{array}$ & $\mathrm{P}$ \\
\hline & Frec (\%) & Frec (\%) & Frec (\%) & Frec (\%) & Frec (\%) & Frec (\%) & \\
\hline Global & $178(100)$ & $23(12.9)$ & $18(10.1)$ & $97(54.7)$ & $23(12.9)$ & $17(9.6)$ & \\
\hline \multicolumn{8}{|l|}{$\begin{array}{l}\text { Sensibilidad } \\
\text { dental }\end{array}$} \\
\hline No & 163(91.6) & $23(100)$ & 16(89.9) & $88(90.7)$ & $20(87.0)$ & $16(94.1)$ & 0.532 \\
\hline $\mathrm{Si}$ & $15(8.4)$ & --- & $2(11.1)$ & $9(9.3)$ & $3(13.0)$ & $1(5.9)$ & \\
\hline \multicolumn{8}{|l|}{ Movilidad Dental } \\
\hline Inexistente & $148(83.2)$ & $23(100)$ & 18(100) & 93(95.9) & $13(56.5)$ & $1(5.9)$ & \\
\hline Grado I & $27(15.2)$ & --- & --- & $3(3.1)$ & $10(43.5)$ & $14(82.2)$ & $<0.000+$ \\
\hline $\begin{array}{l}\text { Grado II } \\
\text { Índice de placa }\end{array}$ & $3(1.7)$ & --- & --- & $1(1.0)$ & --- & $2(11.8)$ & \\
\hline Bueno & $45(25.3)$ & $23(100)$ & 13(72.2) & $8(8.3)$ & $1(4.4)$ & -- & \\
\hline Regular & $73(41.0)$ & --- & $4(22.2)$ & $61(62.9)$ & $6(26.1)$ & $2(11.8)$ & $<0.000+$ \\
\hline Malo & 60(33.7) & --- & $1(5.6)$ & $28(28.9)$ & $16(69.6)$ & $15(88.2)$ & \\
\hline
\end{tabular}

* Test exacto de Fisher 
Tabla 3. Modelo de regresión binomial de enfermedad periodontal.

\begin{tabular}{lccc}
\hline \multicolumn{1}{c}{ Variable } & RR & IC 95\% & P \\
\hline Fuma & 1.97 & $1.05-3.68$ & 0.033 \\
Edad & 1.03 & $1.0-1.1$ & 0.011 \\
Sexo & 0.76 & $0.37-1.53$ & 0.446 \\
Nivel educativo & & & \\
Primaria & 1.87 & --- & 1.0 \\
Secundaria & 0.84 & --- & 1.0 \\
Universidad & 0.62 & --- & 1.0 \\
Higiene oral & & & \\
Regular & 2.77 & $1.79-4.27$ & $<0.001$ \\
Mala & 2.77 & $1.79-4.27$ & $<0.001$ \\
\hline
\end{tabular}

Tabla 4. Modelo de regresión binomial final o ajustado para enfermedad periodontal.

\begin{tabular}{lccc}
\hline \multicolumn{1}{c}{ Variable } & RR & IC 95\% & P \\
\hline Fuma & 3.35 & $1.61-6.95$ & 0.001 \\
Nivel de educación universitario & 0.35 & $0.11-1.14$ & 0.08 \\
Higiene oral & & & \\
Regular & 4.33 & $1.73-10.81$ & 0.002 \\
Mala & 2.77 & --- & - \\
\hline
\end{tabular}

\section{DISCUSIÓN}

El estudio de la relación entre la enfermedad periodontal y el tabaco ha recibido cada vez más atención en los últimos años. Una búsqueda de los trabajos publicados indica que en los últimos cuatro años se han escrito más de 200 artículos sobre el tema. Recientemente, se han realizado excelentes revisiones, ${ }^{20,21}$ que incluyen artículos sobre la postura de la Academia Americana de Periodoncia al respecto. $^{22}$

El tabaquismo es un factor de riesgo importante para muchas enfermedades crónicas que imponen una carga sustancial a la salud general. Desde la perspectiva de la salud oral, el uso del tabaco es uno de los factores de riesgo más significativos para la evolución de la enfermedad periodontal en poblaciones con higiene oral regular ${ }^{23}$.

En este estudio se pudo corroborar que el fumar asociado a un índice de higiene oral regular se relaciona con una alta prevalencia de enfermedad periodontal y la influencia de estos dos factores en forma conjunta se podrían constituir en factores potenciadores para determinar el desarrollo de la enfermedad periodontal.

Adicionalmente, se encontró que casi el $90 \%$ de los sujetos evaluados registraron enfermedad perio- dontal; sin embargo, es importante observar una disminución del sangrado al sondaje en las personas con enfermedad periodontal y que reportaban ser fumadores, similar a los resultados reportados por Preber y Bergstrom, y por Bergstrom y Bostrom citados por Lordelo, ${ }^{24}$ pues al revisar 369 pacientes con periodontitis entre moderada y grave, los fumadores tenían una menor cantidad de áreas sangrantes (25\%) que los no fumadores (51\%), de igual forma apoyaron el argumento de que la respuesta inflamatoria gingival puede estar suprimida en los fumadores. Por otro lado, Mirbod y colaboradores establecieron que cuando se observa la densidad de la vascularización gingival vestibular utilizando anticuerpos monoclonales frente a la molécula CD34 expresada en las células endoteliales y las células progenitoras hematopoyéticas, en los fumadores existe una mayor proporción de pequeños vasos sanguíneos y una menor proporción de grandes vasos, en comparación con los no fumadores, mientras que las diferencias en densidad no son significativas. ${ }^{25}$

En este estudio, la presencia de al menos un marcador periodontal se observó en el (87\%) de los evaluados y la proporción de personas con pérdida de inserción aumenta con la edad; resultados similares a los registrados por el Tercer Estudio Nacional de Salud Bucal del año 1998 (ENSAB III) ${ }^{8}$ que en estudios anteriores sobre la salud periodontal 
de los colombianos en 1966 , el (88.7\%) de la población tenía enfermedades periodontales en cualquiera de sus fases; entre 1977 y 1980, el (94.7\%); y en 1998 el (92.4\%) presentaba por lo menos un parámetro de esta enfermedad (sangrado, cálculos, bolsa) y la proporción de personas con pérdida de inserción periodontal aumentaba a medida que la edad era mayor.

Según el ENSAB III del total de personas incluidas en la valoración periodontal, el $50.2 \%$ presentaron pérdida de inserción, siendo mayor la tasa en hombres $(52.6 \%)$ que en mujeres $(47.6 \%) .{ }^{8} \mathrm{El}(42.0 \%)$ de las personas presentan una pérdida localizada y en el (8.2\%) es generalizada. La pérdida es leve en el (41.1\%) de las personas, en el (7.7\%) es moderado y en el (1.2\%) es severa.

Así mismo, se observó que la extensión y la severidad de la enfermedad ha aumentado en esta población con respecto al ENSAB III (1998) ya que en este estudio el (64\%) de los evaluados presentaron pérdida de inserción y sigue siendo igual con respecto a la pérdida por género; de la misma forma la pérdida sigue siendo más localizada que generalizada y leve.

Estudios realizados por Goultschin y otros (citados por Velasco), ${ }^{26}$ comparan niveles de INTPC en fumadores y no fumadores donde los cálculos y las bolsas de 4 y $5 \mathrm{~mm}$ son prevalentes en fumadores; planteamiento que coincide con los resultados alcanzados por este estudio.

Durante años, los profesionales del cuidado oral han reconocido la importancia de educar a sus pacientes sobre técnicas adecuadas de autocuidado. Se ha probado sin lugar a dudas que la limpieza mecánica regular de los dientes previene la gingivitis, y la mayoría de los estudios sustenta el beneficio de autocuidado para prevenir la caries dental. La prevención de la acumulación de placa, y por lo tanto de gingivitis y caries incluye la autolimpieza diaria de las superficies de fácil acceso con un cepillo de dientes manual o eléctrico y crema dental con flúor, y de las superficies proximales con ayudas como la seda dental o palillo triangulares de madera. ${ }^{27}$

Para la higiene oral, este estudio mostró que los sujetos evaluados presentan en su mayoría índices regulares al igual que la falta de uso de la seda dental; estudios realizados por Axelsson y colaboradores demuestran que educar y motivar a los pacientes resulta efectivo para prevenir caries dental, enfermedad periodontal y pérdida de dientes en adultos. ${ }^{28}$ La negligencia en hábitos de cuidado en salud dental, se ha relacionado con un amplio complejo de problemas en los estilos de vida de los adolescentes; $;^{29,30}$ también, se ha observado que los comportamientos relacionados con salud oral han mostrado estar asociados con fumar, consumo de alcohol y actividad física en la población adulta. ${ }^{31}$

De esta manera, se podría sugerir que los odontólogos deben promover los hábitos de autocuidado no sólo en lo que se refiere a la salud dental si no de forma integral para evitar la adopción del hábito de fumar que se ha considerado de gran importancia en el desarrollo de la enfermedad periodontal. Es necesario usar estrategias para la prevención y el control de la enfermedad periodontal para lograr efectos positivos sobre algunos cuadros sistémicos como enfermedad cardiovascular, cáncer, entre otros.

La comprensión de la relación entre el tabaco y la enfermedad periodontal es el resultado del trabajo de varios grupos de investigadores. Una vez revisada la bibliografía, no puede dejar de mencionarse la significativa contribución al estudio de la enfermedad periodontal y el tabaco del grupo constituido por Bergstrom, Preber y Boström, entre otros, que han estudiado sistemáticamente importantes aspectos de la interacción entre el tabaco y la enfermedad periodontal durante muchos años.

Se podría concluir que el hábito de fumar aunado a una mala higiene oral se pueden constituir en factores muy significativos en el desarrollo y la progresión de las enfermedades periodontales, que el sangrado gingival está disminuido en estos pacientes y que aspectos como la prevención, la educación y la motivación de la higiene oral son fundamentales para el control y evitar que se desarrolle esta patología.

Se sugiere que para próximos estudios sobre el tema se puedan hacer investigaciones microbiológicas e inmunológicas de la placa y el cálculo dental en personas fumadoras. 


\section{BIBLIOGRAFÍA}

1. Genco RJ. Current view of risk factors for periodontal diseases. J. Periodontol 1996. 67: 1041 - 1049.

2. República de Colombia, Ministerio de Salud. Tercer Estudio Nacional de Salud Bucal, ENSAB III, Bogotá. Segundo estudio nacional de factores de riesgo de enfermedades crónicas: Tomo II Tabaquismo. 1999.

3. Grossi SG, Zambon JJ, Ho AW, Koch G, Dunford RG, Machtei EE, Norderyd OM, Genco RJ. Assessment of risk for periodontal disease: Risk indicators for attachment loss. J. Periodontol 1994; 65: 260 - 267.

4. Tomar SL, Asma S. National Health and Nutrition Examination Survey: Smoking attributable periodontitis in the United States. J Periodontol 2000; 71: 743 - 751.

5. Schuller AA, Holst D. An S-Shaped relationship between smoking duration and alveolar bone loss: Generating an hypothesis. J Periodontol 2001; 72: 1164 - 1171.

6. Gaetti-Jardim E, Zanoli T, Pedrini D. O tabagismo como fator de risco para as doenças periodontais: aspectos microbiológicos. Rev Odontol Univ São Paulo 1998. 4: $315-322$.

7. Preber H, Bergström J, Linder LE. Occurrence of periopathogens in smoker and non smoker patients. J Clin Periodontol 1992; 19: 667 - 671.

8. República de Colombia. Ministerio de Salud. III Estudio Nacional de Salud Bucal. II estudio nacional de factores de riesgo de enfermedades crónicas: Tomo VII. 1999.

9. Kelsey JL, Douglas TH. Methods in Obsevational Epidemiology. $1^{\text {a }}$ Ed. New York: Oxford University Press, 1986.

10. Feinstein Alvan R. Clinical biostatistics. The architecture of cross-sectional research (part I). Clin Pharmacol Ther 1978; 23: $81-96$.

11. Feinstein Alvan R. Clinical biostatistics. The architecture of cross-sectional research (Conclusion). Clin Pharmacol Ther 1978; 23: 481-493

12. CDC-OMS. Epi - Inf 6. Versión 6.04. Nov 1996.

13. Microsoft Excel 5.0, Microsoft Corporation 1997.

14. Stata Corp. Stata statistical software. Release 9.1 college station, T.X: Stata corporation.

15. Pagano M, Gauvreau K. Principles of Biostatistics. $1^{\mathrm{a}}$ Ed. Belmont. Duxbury Press. Belmont. p 35-44, 257-263.

16. Norman GR, Streiner Dl. Bioestadística. Mosby/Doyma. Libros. Madrid. 1996 p 129-147.

17. Wacholder S, Binomial regresión in GLIM: Estimating risk and risk differences. Am J Epidemiol 1986; 123: 174 $-184$.

18. Zochetti C, Consonni D, Vertais PA. Relationship between Prevalence Rate Ratios and Odds Ratios in CrossSectional Studies. Int J Epidemiol 1997; 26: 220 - 223.
19. Bautista LE. Métodos avanzados de análisis. Universidad Industrial de Santander. Bucaramanga, 2002.

20. Johnson GK, Slach NA. Impact of tobacco use on periodontal status. J. Dent Educ 2001; 65: 313 -321.

21. Kinane DF, Chertnutt IG. Smoking and periodontal disease. Crit Rev Oral Biol Med 2000; 11: 356 - 365.

22. American Academy of Periodontology. Position Paper: Tobacco use and the periodontal patient: Research, science and therapy committee of the American Academy of Periodontology. J periodontol 1999; 70: 1419 -1427.

23. Axelsson P. External modifying factors involved in periodontal diseases. $1^{\mathrm{a}}$ Ed. Quintessence Publishing Co. 2005.

24. Lordelo MJ. El tabaco y su influencia en el periodonto. Av Periodon Implantol 2005; 171: 17 - 24.

25. Mirbod SM, Ahing SI, Pruthi VK. Immunohistochemical study of vestibular gingival blood vessel density and internal circumference in smokers and non - smokers. J Periodontol 2001; 72: 1318 -1323.

26. Velasco E, Machuca M, Martínez Sabuquillo A, Rios V, Bullon P. Los factores asociados a los trastornos psicológicos y su efecto sobre la cavidad oral. Arch Odontoestom 1994; 7: 382 - 396.

27. Axelsson P. Mechanical plaque control by self-care: Preventive materials, methods. $1^{\mathrm{a}}$ Ed: Quintessence Publishing Co. 2004.

28. Axelsson P, Nyström B, Lindhe J. The long-term effect of a plaque control program on tooth mortality, caries and periodontal disease in adults. Results after 30 years of maintenance. J Clin Periodontol 2004; 31: 749 - 757.

29. Koivusilta L, Honkala S, Honkala E, Rimpelä A. Toothbrushing as part of the adolescent lifestyle predicts educational level. J Dent Res 2003; 82: 361 - 366.

30. Kassak KM, Dagher R, Doughan B. Oral hygiene and lifestyle correlates among new undergraduaduate university students in Lebanon. J Am Coll Health 2001; 50: 15 - 20.

31. Ylöstalo PV, Ek E, Laitinen J, Knuuttila Ml. Optimism and life satisfaction as determinants for dental and general health behavior: Oral health habits linked to cardiovascular risk factors. J Dent Res 2003; 82:194 - 199. 